\title{
Crisp-type fuzzy logic controller using Dubois and Prade's parametric t-norm-sum-gravity inference methods
}

\author{
C.-L.Chen, Y.-C.Lai and C.-T.Hsieh
}

\begin{abstract}
The input/output relationship of a class of crisp-type fuzzy logic controllers (FLCs) using parametric $t$-norm-sum-gravity inference method is examined. The explicit mathematical form of reasoning surface using Dubois and Prade's parametric $t$-norm is addressed. Reasoning surfaces of crisp-type FLCs are proved to be composed of a two-dimensional multilevel relay and a local position-dependent nonlinear compensator. The influence of the $\alpha$ value, the parameter of Dubois and Prade's parametric $t$-norm, on the reasoning surfaces is also investigated.
\end{abstract}

\section{Introduction}

Much emphasis has been placed on the field of fuzzy logic controllers (FLC) since the introduction of fuzzy sets [1]. Owing to the intrinsic parallels between reasoning procedures of FLCs and those of the human operators, FLCs stand out as a viable alternative to those processes that are difficult to control.

Since the advance of the first FLC proposed by Mamdani [2], there have been numerous applications of FLCs suggested in almost every field from water quality control to automobile transmission control, and from fuzzy memory devices to fuzzy computers (see [3]). Most of the applications are, however, based on intuitive implementation of domain experts' experiences rather than systematic derivation of the operators used in FLCs. This approach complies with the very concept 'fuzziness' of fuzzy logic, but some important features of FLCs are thus concealed and cannot be gleaned by the designers. Furthermore, theoretical analysis of the basic properties of fuzzy control systems are usually considered as impossible or impractical. This prevailing notion makes the theoretical analysis of FLCs appear to be trivial work for designing FLCs and is not reversed until recently.

A thorough review of the design of FLCs and a solid collection of mathematical analyses for FLCs from a theoretical perspective are given in [3-6]. In [4, 7-9], significant efforts are made in performing the stability analysis and the design principles of fuzzy control systems. Meanwhile, some appealing FLC analyses are also reported in turn, such as [10-15]. These mathematical

\section{(C) IEE 2000}

IEE Proceedings online no. 20000180

DOI: 10.1049/ip-cta:20000180

Paper first received 31st March and in revised form 25th November 1999 The authors are with the Department of Chemical Engineering, National Taiwan University, Taipei 10617, Taiwan, Republic of China analyses clearly describe input/output relations of FLCs under some specific restrictions of membership functions, rule base and inference methods. However, the significance of these investigations is downgraded by some impractical premises proposed in them. For example, all the membership functions adopted in $[10,13-15]$ are equally spaced triangles. However, it has been shown that the control ability of an FLC equipped with uniformly distributed triangular membership functions is inadequate for complex systems $[10,11]$. On the other hand, in $[11,12]$, the restriction of membership functions has been chosen to include unequally spaced triangles. However, the inference operator is based only on the so-called product-sum-gravity method, and the analyses have been focused on the similarity and difference between the FLC and the linear PI controller. Recently, Chen et al. [4, 16] have extended the FLC analyses to adopt arbitrarily distributed triangular fuzzy partitions and to apply four frequently used $t$ norms for inference.

This article aims to generalise the theoretical analyses given in previous related researches $[4,10,12-16]$. Therein, the crisp-type output membership functions and the triangular-type input fuzzy partitions can be nonuniformly spaced, and the Dubois and Prade's parametric $t$-norm is applied, as an illustration, in evaluating true values of the control rules. The parametric $t$-norms, although not widely implemented in practical control procedures, possess the merits that they can be turned into nearly all types of nonparametric t-norms used in applications such as the standard fuzzy intersection (the minimum) and/or the algebraic product by only modifying their parameters. This feature is advantageous to both the FLC designer and field operators, because they can alter the behaviour of the FLC without first changing its operators. The explicit input/output relations for the FLCs using the Dubois and Prade's parametric $t$-norm-sum-gravity inference methods will be derived in this paper. The influence of applying various $\alpha$ values in the Dubois and Prade's parametric $t$-norm for inference on output patterns will be studied in this paper. It is expected that these analyses can provide some insight for effective FLC design and tuning. 


\section{Architecture of the fuzzy logic controllers}

As the architecture of the FLC used in the following derivation is the same as that of [16], only an essential description is included here.

For an FLC with two inputs (error $e$ and change in error $r$ ) and one output (change in controller output $u$ ), the associative universes of discourse for $e, r, u$ are all set to be $[-1,1]$ for convenience, and therefore $e_{m}$ and $r_{m}$, the sensored values, are converted to $e^{*}$ and $r^{*}$, the actual input values of the FLC, by scaling factors GE and GR. Fig. 1 shows the structure of an FLC and its detailed computational steps [16].

Three term sets, $T_{e}=\left\{E_{i} \mid i \in I_{m_{e}}\right\}, T_{r}=\left\{R_{j} \mid j \in I_{m_{e}}\right\}$ and $T_{u}=\left\{U_{k} \mid k \in I_{m_{u}}\right\}$, consisting of sets of linguistic terms, $E_{i}$, $R_{j}$ and $U_{k}$, individually, are used to denote the possible linguistic values for $e, r$ and $u ; I_{m_{*}}=\left\{-m_{*}, \ldots,-1,0\right.$, $\left.1, \ldots, m_{*}\right\}$ is the index set.

The input membership functions, $E_{i}(e)$ and $R_{j}(r)$, are triangular fuzzy partitions, i.e. $\Sigma_{\forall i} E_{i}(e)=1, \forall e \in U_{e}$ and $\Sigma_{\forall j} R_{j}(r)=1, \forall r \in U_{r}$. For $E_{i}(e)$, the explicit functions are defined as

$$
\begin{aligned}
E_{i}(e)= & \Lambda\left(e ; E_{i-1}^{*}, E_{i}^{*}, E_{i+1}^{*}\right) \\
= & \left\{\begin{array}{cl}
\frac{e-E_{i-1}^{*}}{E_{i}^{*}-E_{i-1}^{*}} & \text { for } E_{i-1}^{*} \leq e \leq E_{i}^{*} \\
\frac{E_{i+1}^{*}-e}{E_{i+1}^{*}-E_{i}^{*}} & \text { for } E_{i}^{*} \leq e \leq E_{i+1}^{*} \\
0 & \text { otherwise }
\end{array} \text { for } i \in I_{m_{e}}\right.
\end{aligned}
$$

where $E_{i}^{*}$ are principal values with the property that $-1=E_{-m_{e}}^{*}<\ldots<E_{-1}^{*}<E_{0}^{*}=0<E_{1}^{*}<\cdots<E_{m_{e}}^{*}=1$. $R_{j}^{*}$ and $R_{j}(r)$ are defined in similar ways.

As for the output membership functions $U_{k}(u)$, the crisptype membership functions (singleton) are considered. Let $-1=U_{-m_{u}}^{*}<\cdots<U_{-1}^{*}<U_{0}^{*}=0<U_{1}^{*}<\cdots<U_{m_{u}}^{*}=1$, then

$$
U_{k}(u)= \begin{cases}1 & \text { for } u=U_{k}^{*} \\ 0 & \text { otherwise }\end{cases}
$$

The assignment of principal values can be performed arbitrarily as long as the given conditions are observed. This type of membership functions (for either triangular fuzzy partitions or fuzzy singletons) is denoted as unequalspan membership functions (USMFs). In addition to the USMFs, two types of systematically allocated membership functions are also used in this research to derive some important properties. The first type is the shrinking-span membership function (SSMF) [17], in which the principal values are defined as

$$
E_{i}^{*}=\frac{i}{m_{e}} s_{e}^{m_{e}-|i|}, \quad R_{j}^{*}=\frac{j}{m_{r}} s_{r}^{m_{r}-|j|}, \quad U_{k}^{*}=\frac{k}{m_{u}} s_{u}^{m_{u}-|k|}
$$

where $s_{e}, s_{r}, s_{u} \in(0,1]$ are the shrinking factors for $e, r$ and $u$, respectively. If the shrinking factors are all set to unity, then equal-span membership functions (ESMFs) are obtained and the distance between any two neighbouring principal values of the ESMFs is equal to the reciprocal of the respective $m$. The notations USMFs-, SSMFs-, ESMFsFLC are used to indicate the types of membership functions used in the FLC.

The control rule in the rule base has the form

$$
\begin{gathered}
R_{i, j}: \text { IF } e \text { is } E_{i} \text { AND } r \text { is } R_{j} \text { THEN } u \text { is } U_{k=f(i, j)} \\
\forall i \in I_{m_{e}}, j \in I_{m_{r}}
\end{gathered}
$$

For simple rule base, $f(i, j)=i+j$.

In the decision-making step of FLC, under the assumption that $m_{e}=m_{r}=m$, given input pattern $e^{*}$ and $r^{*}$, a series of fuzzy operators, $\mathbb{F}, \mathbb{\square}, \mathbb{A}$ and $\mathbb{D}$ are used to obtain the final output action [17]:

$$
u^{*}=\mathbb{D}\left(\underset{\forall i, j \in I_{m}}{\mathbb{A}}\left\{\left[\left[\mathbb{F}\left(E_{i}\left(e^{*}\right), R_{j}\left(r^{*}\right)\right), U_{f(i, j)}(u)\right]\right\}\right)\right.
$$

The $\mathbb{F}$ calculates the firing level, $\phi_{i, j}=\mathbb{F}\left(E_{i}\left(e^{*}\right), R_{j}\left(r^{*}\right)\right)$, by using $t$-norm operator for each rule in the rule base; $\square$ and A perform the implication and aggregation of the FLC, respectively; and $\mathbb{D}$ converts the aggregated output fuzzy set $U(u)$ into a single crisp output value $u^{*}$. In this article, the authors have chosen the summation for $\mathbb{A}$, the centre of area (COA) method for $\mathbb{D}$, and the algebraic product for $\mathbb{0}$. However, as the output membership functions are fuzzy singletons, the choice of $\rrbracket$ is irrelevant to the final inference result. As for the $\mathbb{F}$ operator, the Dubois and Prade's parametric $t$-norm described in Section 3 is employed to find the firing levels. This type of fuzzy controllers is named the crisp-type FLCs using parametric $t$-norm-sumgravity inference methods by the authors. The final crisp output $u_{m}$ for such crisp-type FLCs is $[12,13]$ :

$$
u_{m}=\mathrm{GU} \times u^{*}=\mathrm{GU} \times \frac{\sum_{\phi_{i, j} \neq 0} \phi_{i, j} U_{f(i, j)}^{*}}{\sum_{\phi_{i, j} \neq 0} \phi_{i, j}}
$$

According to the properties of triangular fuzzy partitions used in the USMFs-FLCs, at most four control rules are fired in the $i j$ th block, i.e. for input pattern $e^{*} \in\left[E_{i}^{*}, E_{i+1}^{*}\right]$ and $r^{*} \in\left[R_{j}^{*}, R_{j+1}^{*}\right]\left(i, j \in I_{m}-\{m\}\right)$, there are at most four

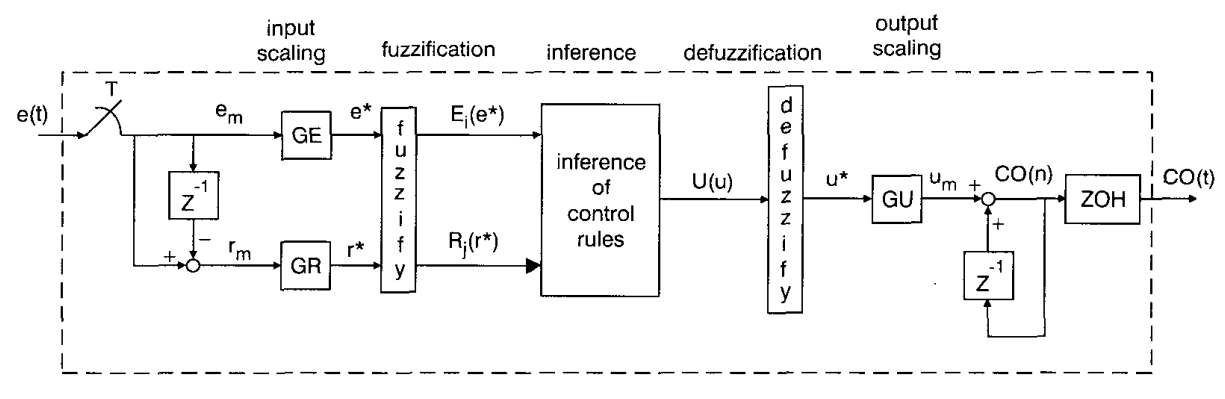

Fig. 1 Detailed computational steps of fuzzy logic controller 
rules having nonzero firing levels $[4,13,16]$. These four rules are as follows:

(i) if $e$ is $E_{i+1}$ and $r$ is $R_{j+1}$ then $u$ is $U_{f(i+1, j+1)}$;

(ii) if $e$ is $E_{i+1}$ and $r$ is $R_{j}$ then $u$ is $U_{f(i+1, j)}$;

(iii) if $e$ is $E_{i}$ and $r$ is $R_{j+1}$ then $u$ is $U_{f(i, j+1)}$;

(iv) if $e$ is $E_{i}$ and $r$ is $R_{j}$ then $u$ is $U_{f(i, j)}$.

Hence, it is straightforward, from eqn. 4 , that $u^{*}$ is bounded by the maximal and minimum of the four centroids, and, for simple rule base, it is

$$
U_{i+j}^{*} \leq u^{*} \leq U_{i+j+2}^{*}
$$

The details of the input/output relations of these FLCs will be described in the following Section.

\section{Mathematical input-output relation of the FLC}

According to eqn. 4, the final control output cannot be obtained without first acquiring the firing levels. Generally speaking, the $t$-norms used to find the firing levels in practical applications are, for their simplicity, either fuzzy intersection (min) or algebraic product. However, the parametric $t$-norms, although not widely implemented in practical control procedures, possess the merits that they can be turned into nearly all types of nonparametric $t$ norms used in industries by only adjusting their parameters.

Many classes of parametric $t$-norms have been proposed to perform fuzzy intersections of various strengths, such as Hamacher, Frank, Dubois and Prade, Yager, and Weber, to name just a few (see [18] for a list of main families of parametric $t$-norms with detailed discussions). Without loss of generality, the authors have chosen the Dubois and Prade's parametric $t$-norm for subsequent analysis because of its simplicity (it has simple functional form with bounded parameter range) and flexibility (it covers the whole useful range of $t$-norms with algebraic product and standard intersection as its lower bound and upper bound, respectively). Notably, it is possible to conduct similar examination by using other simple parametric $t$-norms such as Weber or Hamacher families.

This Section provides a clear derivation and some important properties for the USMFs-FLCs using the Dubois and Prade's parametric $t$-norm-sum-gravity inference methods.

Because some common features are shared by all $i j$ th blocks, it is more convenient to defined a normalised $i j$ th block, a unit square, by setting the normalised input values $\varepsilon=\left(e^{*}-E_{i}^{*}\right) /\left(E_{i+1}^{*}-E_{i}^{*}\right)$ and $\mathcal{R}=\left(r^{*}-R_{j}^{*}\right) /\left(R_{j+1}^{*}-R_{j}^{*}\right)$ than using $e^{*}, r^{*}$ alone. And hence, for input pattern $\left(e^{*}, r^{*}\right) \in i j$ th block, the four nonzero membership values are $\quad E_{i+1}\left(e^{*}\right)=\varepsilon, \quad E_{i}\left(e^{*}\right)=1-\varepsilon, \quad R_{j+1}\left(r^{*}\right)=\mathcal{R}, \quad$ and $R_{j}\left(r^{*}\right)=1-\mathcal{R}$.

In order to find the firing levels listed in eqn. 4 , a $t$-norm operator must be choose to perform this task. Here, for reasons stated in the beginning of this Section, the authors use the Dubois and Prade's parametric $t$-norm defined below as the fuzzy intersection operator (see [18]):

$$
\begin{aligned}
& \underset{D \& P}{T}(a, b, \alpha)=\frac{a \cdot b}{\max (a, b, \alpha)} \quad \alpha \in[0,1]
\end{aligned}
$$

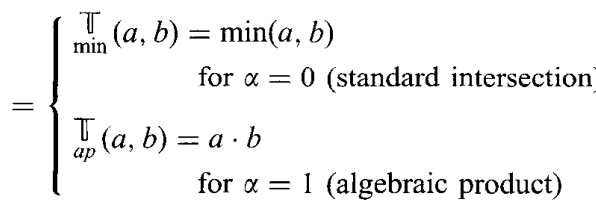

Notably, $\mathbb{T}_{a p}\left(a, b \leq \mathbb{T}_{D \& P}(a, b) \leq \mathbb{T}_{\min }(a, b)\right.$. The firing levels of the four rules listed in the preceding Section are, therefore:

$$
\begin{aligned}
\phi_{i+1, j+1} & =\underset{D \& P}{\mathbb{T}}(\varepsilon, \mathcal{R}, \alpha) & \phi_{i+1, j} & =\underset{D \& P}{\mathbb{T}}(\varepsilon, 1-\mathcal{R}, \alpha) \\
\phi_{i, j+1} & =\underset{D \& P}{\mathbb{T}}(1-\varepsilon, \mathcal{R}, \alpha) & \phi_{i, j} & =\underset{D \& P}{\mathbb{T}}(1-\varepsilon, 1-\mathcal{R}, \alpha)
\end{aligned}
$$

Owing to the max operator shown in the equation for $\mathbb{T}_{D \& P}$, the relative magnitudes of $\varepsilon, \mathcal{R}$ and $\alpha$ determine the inference results and, therefore, some partitions are made in the three-dimensional space constituted by $\varepsilon, \mathcal{R}$ and $\alpha$ to emphasise the relationship among them.

For variables $(\varepsilon, \mathcal{R})$, it has been shown that $[4,16]$ there are four different combinations of the magnitudes of $(\varepsilon, \mathcal{R})$, and these four regions are designated as regions $1 \sim 4$ as shown in Fig. 2. The same partitions can be applied to $(\mathcal{R}$, $\alpha)$ and $(\varepsilon, \alpha)$, and the relative magnitude of $\varepsilon, \mathcal{R}$ and $\alpha$ in these regions are all shown in Table 1 .

Because the range of the three variables are all of $[0,1]$, a unit cube is formed by applying the three variables as three co-ordinates. Slicing the unit cube along the two diagonal lines on $\varepsilon-\mathcal{R}-0$ plane, $0-\mathcal{R}-\alpha$ plane and $\varepsilon-0-\alpha$ plane, respectively, as can be shown in Fig. 3, 24 triangular cones are obtained.

All points in any one of the 24 cones have the same relative magnitude among $\varepsilon, \mathcal{R}$ and $\alpha$, and therefore all input values located in a specific cone would have the same
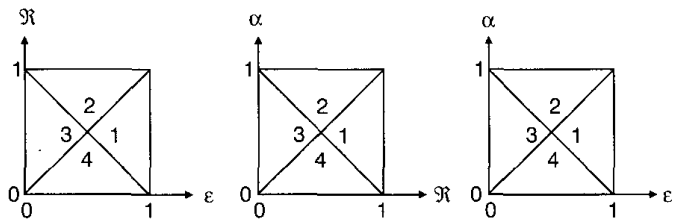

a
Fig. 2 Block diagram showing the four designated regions shown in Table 1

a $\approx-\mathcal{R}$ block

$b \mathcal{R}-\alpha$ block

$c \varepsilon-\alpha$ block

Table 1: Relative magnitude of $\varepsilon, \mathcal{R}$ and $\alpha$ on $\varepsilon-\mathcal{R}-0$ plane, $0-\mathcal{R}-\alpha$ plane, and $\varepsilon-0-\alpha$ plane, respectively

\begin{tabular}{llll}
\hline Region & $\varepsilon-\mathcal{R}$ block & $\mathcal{R}$ - $\alpha$ block & $\varepsilon-\alpha$ block \\
\hline 1 & $\varepsilon \geq \mathcal{R}$ & $\mathcal{R} \geq \alpha$ & $\varepsilon \geq \alpha$ \\
& $(1-\mathcal{R} \geq 1-\varepsilon)$ & & \\
& $\varepsilon \geq 1-\mathcal{R}$ & $\alpha \geq 1-\mathcal{R}$ & $\alpha \geq 1-\varepsilon$ \\
& $(\mathcal{R} \geq 1-\varepsilon)$ & & \\
& $\mathcal{R} \geq \varepsilon$ & $\alpha \geq \varepsilon$ \\
& $(1-\varepsilon \geq 1-\mathcal{R})$ & & \\
& $\varepsilon \geq 1-\mathcal{R}$ & $\alpha \geq 1-\mathcal{R}$ & $\alpha \geq 1-\varepsilon$ \\
& $(\mathcal{R} \geq 1-\varepsilon)$ & & \\
& $\mathcal{R} \geq \varepsilon$ & $\alpha \geq \varepsilon$ \\
& $(1-\varepsilon \geq 1-\mathcal{R})$ & & \\
& $1-\mathcal{R} \geq \varepsilon$ & $1-\mathcal{R} \geq \alpha$ & $1-\varepsilon \geq \alpha$ \\
& $(1-\varepsilon \geq \mathcal{R})$ & & \\
& $\varepsilon \geq \mathcal{R}$ & $\mathcal{R} \geq \alpha$ & $\varepsilon \geq \alpha$ \\
& $(1-\mathcal{R} \geq 1-\varepsilon)$ & & \\
4 & $1-\mathcal{R} \geq \varepsilon$ & $1-\mathcal{R} \geq \alpha$ & $1-\varepsilon \geq \alpha$ \\
& $(1-\varepsilon \geq \mathcal{R})$ & & \\
\hline
\end{tabular}


inference results for all four rules fired. Fig. 4 depicts the four cones with base on the $\varepsilon-\mathcal{R}-0$ plane, denoted as $c_{i 44}$, $i \in\{1,2,3,4\}$. Subscripts $i, j$ and $k$ of the cone $c_{i j k}$ mean that the projection of the points of the cone would fall on region $i$ of the $\varepsilon-\mathcal{R}-0$ plane, region $j$ of the $0-\mathcal{R}-\alpha$ plane and region $k$ of the $\varepsilon-0-\alpha$ plane, respectively.

For the normalised inputs $\varepsilon$ and $\mathcal{R}$, the firing levels of the four rules within each of these 24 cones are shown in Table 2. By applying the firing levels listed in Table 2, the explicit mathematical form of final output $\mathrm{GU} \times u^{*}$ of the USMFs-FLC, under the assumption of simple rule base, can be expressed as the sum of a global part, $\mathrm{GU} \times u_{G}^{*}$, and a local part, $\mathrm{GU} \times u_{L}^{*}$.
The value of the global part of the crisp output, regardless of the $\alpha$ value, is determined by the specific $i j$ th block in which $\left(e^{*}, r^{*}\right)$ is located and is a constant across the four regions within the $i j$ th block. However, as can be shown in Theorem 1, the local part varies with the values of $e^{*}, r^{*}$ and $\alpha$.

Theorem 1: For the crisp-type USMFs-FLC using the Dubois and Prade's parametric $t$-norm-sum-gravity inference method and simple rule base, let $\varepsilon=\left(e^{*}-E_{i}^{*}\right) /$ $\left(E_{j+1}^{*}-E_{i}^{*}\right)$ and $\mathcal{R}=\left(r^{*}-R_{j}^{*}\right) /\left(R_{j+1}^{*}-R_{j}^{*}\right)$ denote the normalised input values for any given input pattern in the $i j$ th block, i.e. $e^{*} \in\left[E_{i}^{*}, E_{i+1}^{*}\right]$ and $r^{*} \in\left[E_{j}^{*}, R_{j+1}^{*}\right]$, the explicit input/output relation is the sum of a global two- $\alpha$

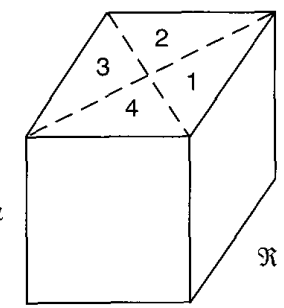

$\varepsilon$

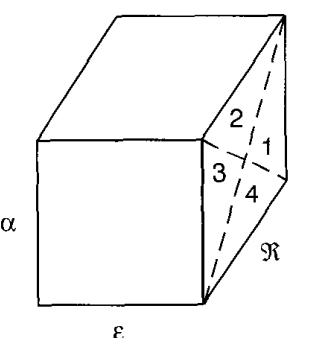

$\varepsilon$

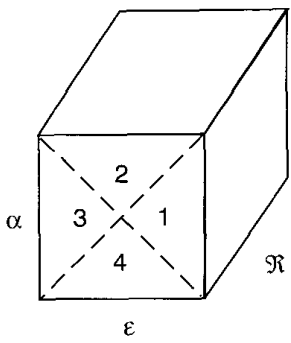

Fig. 3 Slicing of $\varepsilon-\mathcal{R}-\alpha$ unit cube
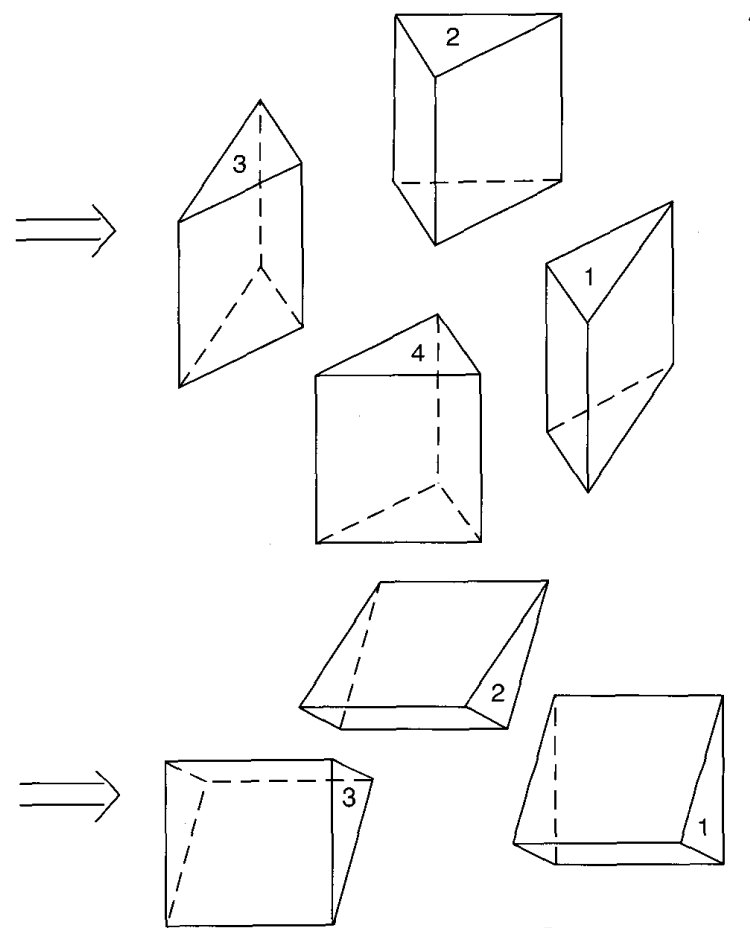

common

intersecting

line
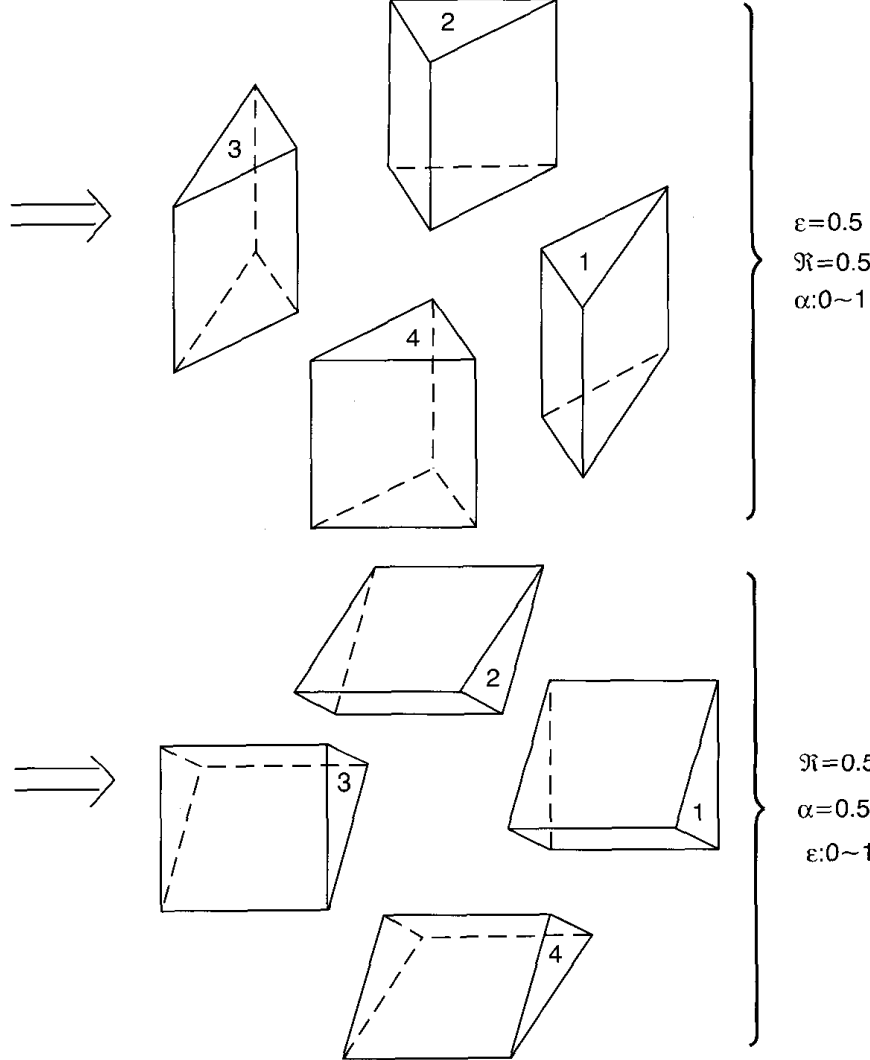

$\Re=0.5$

$\alpha=0.5$

$\varepsilon: 0 \sim 1$ cones 

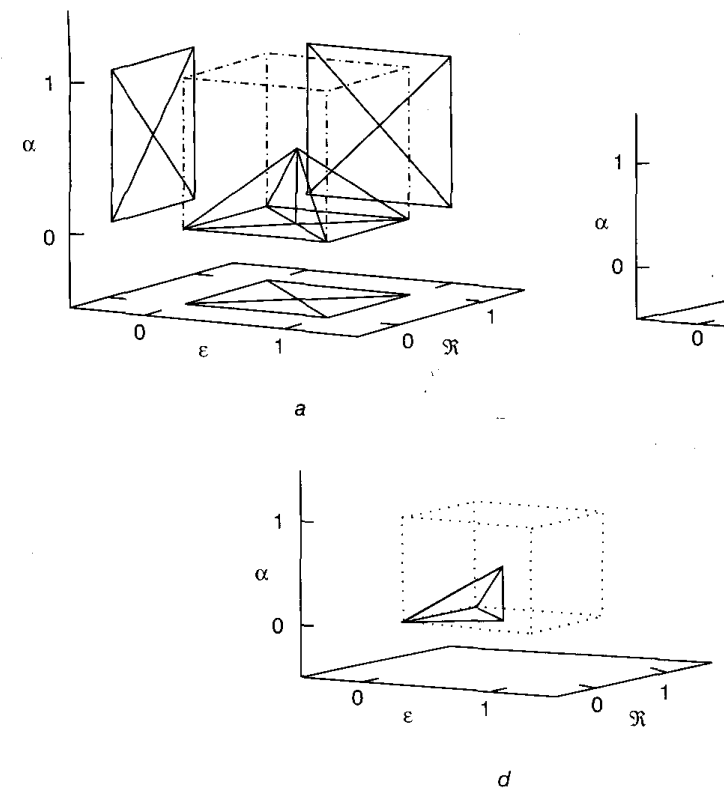
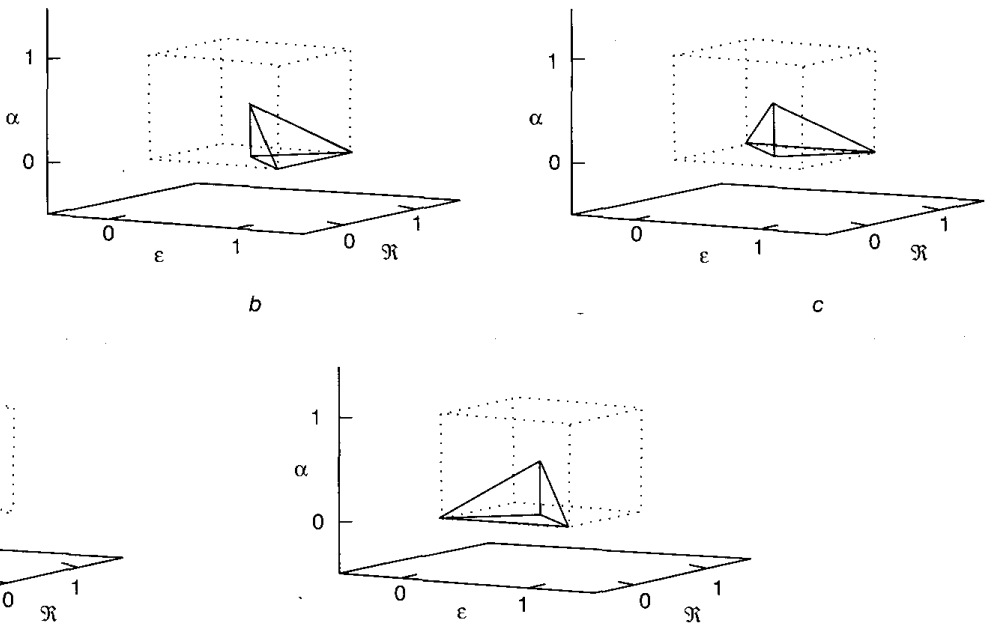

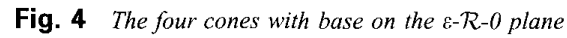

$a$ The $\varepsilon-\mathcal{R}-0$ plane

b $c_{144}$

$c c_{244}$

$d c_{344}$
$e c_{444}$

Table 2: Firing levels of each rule in the 24 cones

\begin{tabular}{|c|c|c|c|c|c|c|}
\hline \multirow[t]{2}{*}{ Based on } & \multirow[t]{2}{*}{ Region } & \multicolumn{4}{|c|}{ Firing level of each rule } & \multirow[t]{2}{*}{ Summation of firing levels } \\
\hline & & rule 1 & rule 2 & rule 3 & rule 4 & \\
\hline & & $\phi_{i+1, j+1}$ & $\phi_{i+1, j}$ & $\phi_{i, j+1}$ & $\phi_{i, j}$ & $\sum_{\phi_{i, j} \neq 0} \phi_{i, j}$ \\
\hline & $c_{144}$ & $\mathcal{R}$ & $1-\mathcal{R}$ & $1-\varepsilon$ & $1-\varepsilon$ & $1+2(1-\varepsilon)$ \\
\hline & $c_{244}$ & $\varepsilon$ & $1-\mathcal{R}$ & $1-\varepsilon$ & $1-\mathcal{R}$ & $1+2(1-\mathcal{R})$ \\
\hline \multirow[t]{4}{*}{$\varepsilon-\mathcal{R}-0$} & $c_{344}$ & $\varepsilon$ & $\varepsilon$ & $\mathcal{R}$ & $1-\mathcal{R}$ & $1+2 \varepsilon$ \\
\hline & $c_{444}$ & $\mathcal{R}$ & $\varepsilon$ & $\mathcal{R}$ & $1-\varepsilon$ & $1+2 \mathcal{R}$ \\
\hline & $c_{122}$ & $\varepsilon \mathcal{R} / \alpha$ & $\varepsilon(1-\mathcal{R}) / \alpha$ & $(1-\varepsilon) \mathcal{R} / \alpha$ & $(1-\varepsilon)(1-\mathcal{R}) / \alpha$ & $1 / \alpha$ \\
\hline & $c_{222}$ & $\varepsilon \mathcal{R} / \alpha$ & $\varepsilon(1-\mathcal{R}) / \alpha$ & $(1-\varepsilon) \mathcal{R} / \alpha$ & $(1-\varepsilon)(1-\mathcal{R}) / \alpha$ & $1 / \alpha$ \\
\hline \multirow[t]{4}{*}{$\varepsilon-\mathcal{R}-1$} & $c_{322}$ & $\varepsilon \mathcal{R} / \alpha$ & $\varepsilon(1-\mathcal{R}) / \alpha$ & $(1-\varepsilon) \mathcal{R} / \alpha$ & $(1-\varepsilon)(1-\mathcal{R}) / \alpha$ & $1 / \alpha$ \\
\hline & $c_{422}$ & $\varepsilon \mathcal{R} / \alpha$ & $\varepsilon(1-\mathcal{R}) / \alpha$ & $(1-\varepsilon) \mathcal{R} / \alpha$ & $(1-\varepsilon)(1-\mathcal{R}) / \alpha$ & $1 / \alpha$ \\
\hline & $c_{313}$ & $\varepsilon$ & $a(1-\mathcal{R}) / \alpha$ & $\mathcal{R}$ & $1-\mathcal{R}$ & $1+\varepsilon+\varepsilon(1-\mathcal{R}) / \alpha$ \\
\hline & $c_{323}$ & $\varepsilon \mathcal{R} / \alpha$ & $\varepsilon(1-\mathcal{R}) / \alpha$ & $\mathcal{R}$ & $1-\mathcal{R}$ & $1+\varepsilon / \alpha$ \\
\hline \multirow[t]{4}{*}{$0-\mathcal{R}-\alpha$} & $c_{333}$ & $\varepsilon \mathcal{R} / \alpha$ & $\varepsilon$ & $\mathcal{R}$ & $1-\mathcal{R}$ & $1+\varepsilon+\varepsilon \mathcal{R} / \alpha$ \\
\hline & $c_{343}$ & $\varepsilon$ & $\varepsilon$ & $\mathcal{R}$ & $1-\mathcal{R}$ & $1+2 \varepsilon$ \\
\hline & $c_{111}$ & $\mathcal{R}$ & $1-\mathcal{R}$ & $1-\varepsilon$ & $(1-\varepsilon)(1-\mathcal{R}) / \alpha$ & $1+(1-\varepsilon)+(1-\varepsilon)(1-\mathcal{R}) / \alpha$ \\
\hline & $c_{121}$ & $\mathcal{R}$ & $1-\mathcal{R}$ & $(1-\varepsilon) \mathcal{R} / \alpha$ & $(1-\varepsilon)(1-\mathcal{R}) / \alpha$ & $1+(1-\varepsilon) / \alpha$ \\
\hline \multirow[t]{4}{*}{$1-\mathcal{R}-\alpha$} & $c_{131}$ & $\mathcal{R}$ & $1-\mathcal{R}$ & $(1-\varepsilon) \mathcal{R} / \alpha$ & $1-\varepsilon$ & $1+(1-\varepsilon)+(1-\varepsilon) \mathcal{R} / \alpha$ \\
\hline & $c_{141}$ & $\mathcal{R}$ & $1-\mathcal{R}$ & $1-\varepsilon$ & $1-\varepsilon$ & $1+2(1-\varepsilon)$ \\
\hline & $c_{431}$ & $\mathcal{R}$ & $\varepsilon$ & $(1-\epsilon) \mathcal{R} / \alpha$ & $1-\varepsilon$ & $1+\mathcal{R}+(1-\varepsilon) \mathcal{R} / \alpha$ \\
\hline & $c_{432}$ & $\varepsilon \mathcal{R} / \alpha$ & $\varepsilon$ & $(1-\varepsilon) \mathcal{R} / \alpha$ & $1-\varepsilon$ & $1+\mathcal{R} / \alpha$ \\
\hline \multirow[t]{4}{*}{$8-0-\alpha$} & $c_{433}$ & $\varepsilon \mathcal{R} / \alpha$ & $\varepsilon$ & $\mathcal{R}$ & $1-\varepsilon$ & $1+\mathcal{R}+\varepsilon \mathcal{R} / \alpha$ \\
\hline & $c_{434}$ & $\mathcal{R}$ & $\varepsilon$ & $\mathcal{R}$ & $1-\varepsilon$ & $1+2 \mathcal{R}$ \\
\hline & $c_{211}$ & $\varepsilon$ & $1-\mathcal{R}$ & $1-\varepsilon$ & $(1-\varepsilon)(1-\mathcal{R}) / \alpha$ & $1+(1-\mathcal{R})+(1-\epsilon)(1-\mathcal{R}) / \alpha$ \\
\hline & $c_{212}$ & $\varepsilon$ & $\varepsilon(1-\mathcal{R}) / \alpha$ & $1-\varepsilon$ & $(1-\hat{\varepsilon})(1-\mathcal{R}) / \alpha$ & $1+(1-\mathcal{R}) / \alpha$ \\
\hline \multirow[t]{2}{*}{$\varepsilon-1-\alpha$} & $c_{213}$ & $\varepsilon$ & $\varepsilon(1-\mathcal{R}) / \alpha$ & $1-\varepsilon$ & $1-\mathcal{R}$ & $1+(1-\mathcal{R})+\varepsilon(1-\mathcal{R}) / \alpha$ \\
\hline & $c_{214}$ & $\varepsilon$ & $1-\mathcal{R}$ & $1-\varepsilon$ & $1-\mathcal{R}$ & $1+2(1-\mathcal{R})$ \\
\hline
\end{tabular}




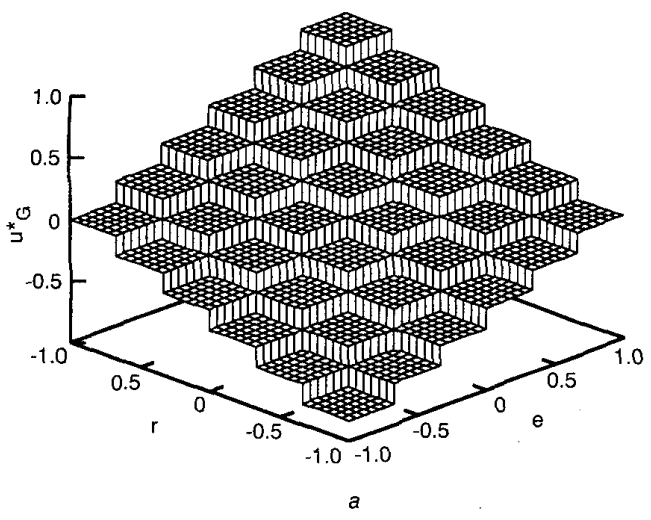

dimensional multilevel relay and an $\alpha$-value dependent local nonlinear compensator:

$$
\begin{aligned}
& u_{m}=\mathrm{GU} \times u^{*}=\mathrm{GU} \times\left(u_{G}^{*}+u_{L}^{*}\right)=u_{G}+u_{L} \\
& u_{G}^{*}=U_{k+1}^{*}
\end{aligned}
$$

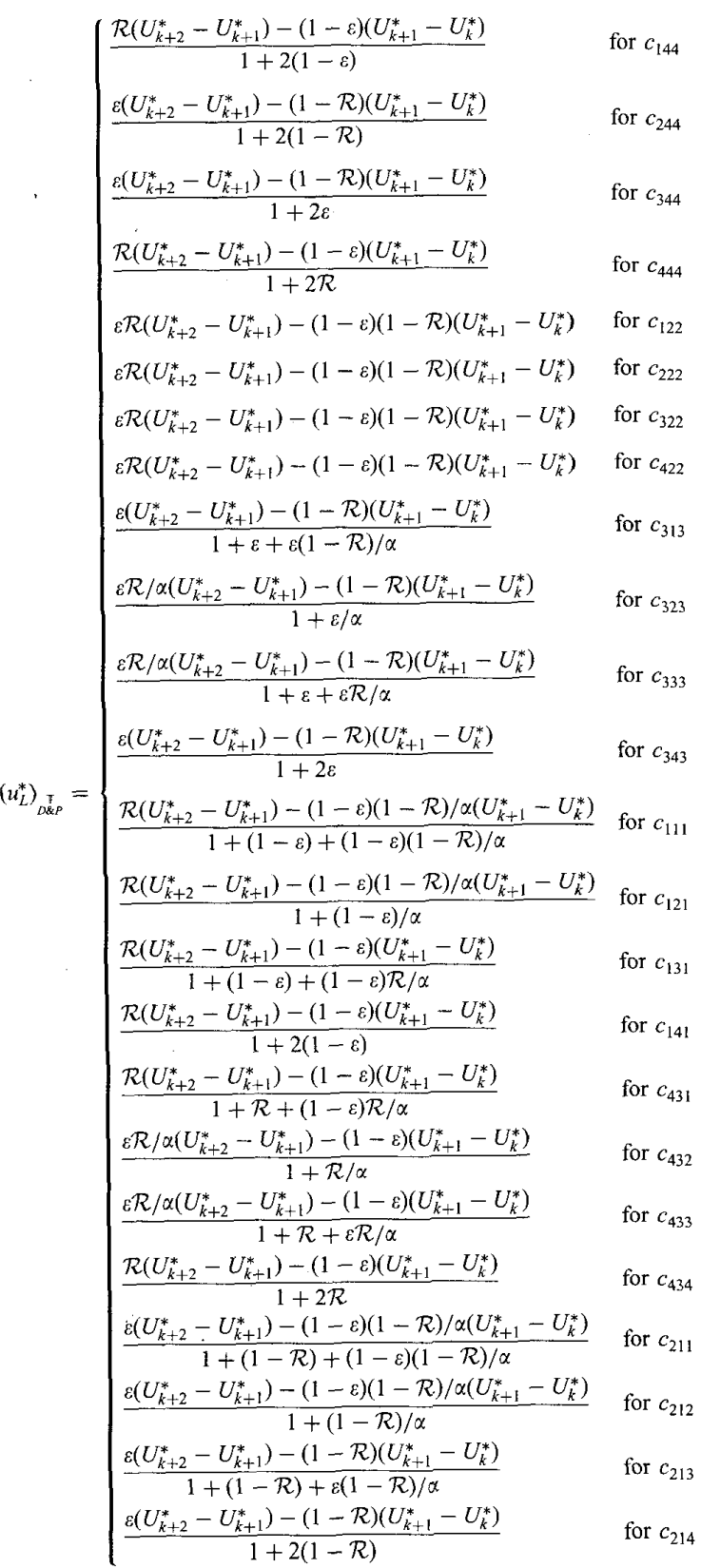

Proof: From eqn. 4 ,

$$
\begin{aligned}
u^{*}= & \frac{\sum_{\phi_{i, j} \neq 0} \phi_{i, j} U_{i+j}^{*}}{\sum_{\phi_{i, j} \neq 0} \phi_{i, j}} \\
= & \frac{\phi_{i+1, j+1} U_{i+j+2}^{*}+\left(\phi_{i+1, j}+\phi_{i, j+1}\right) U_{i+j+1}^{*}+\phi_{i, j} U_{i+j}^{*}}{\phi_{i+1, j+1}+\left(\phi_{i+1, j}+\phi_{i, j+1}\right)+\phi_{i, j}} \\
= & U_{i+j+1}^{*} \\
& +\frac{\phi_{i+1, j+1}\left[U_{i+j+2}^{*}-U_{i+j+1}^{*}\right]-\phi_{i, j}\left[U_{i+j+1}^{*}-U_{i+j}^{*}\right]}{\phi_{i+1, j+1}+\left(\phi_{i+1, j}+\phi_{i, j+1}\right)+\phi_{i, j}}
\end{aligned}
$$

IEE Proc.-Control Theory Appl., Vol. 147, No. 2, March 2000

Fig. 5 Effects of shriking factors on the t-norm-independent global output surface of the crisp-type SSMFs-FLC $\left(m_{e}=m_{r}=3\right)$

$a s_{\mathrm{e}}=s_{\mathrm{r}}=1.0, s_{\mathrm{u}}=1.0$

$b s_{\mathrm{c}}=s_{\mathrm{r}}=0.6, s_{\mathrm{u}}=1.0$

$\begin{aligned} c s_{\mathrm{e}} & =s_{\mathrm{r}}=1.0, s_{\mathrm{u}}=0.6 \\ d s_{\mathrm{e}} & =s_{\mathrm{r}}=0.6, s_{\mathrm{u}}=0.6\end{aligned}$

172 

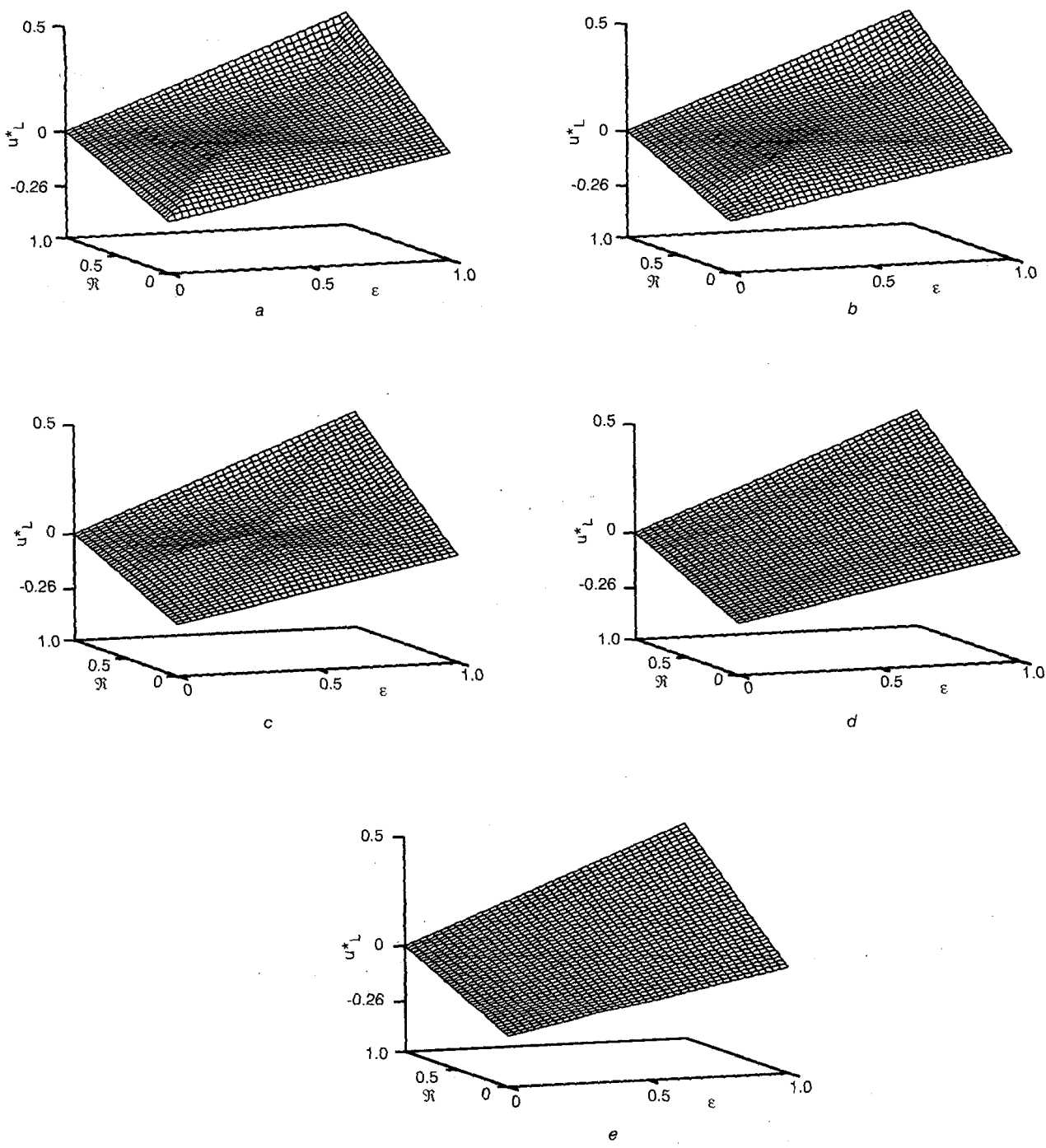

Fig. 6 Illustration for influence of t-norms on local outputs of the crisp-type SSMFs-FLCs $\left(m_{e}=m_{r}=3\right)$

$a \alpha=0$

$b \alpha=0.25$

$c \alpha=0.5$

$d \alpha=0.75$
$e \alpha=1$

Substituting the firing levels for all cones listed in Table 2 leads to the results.

\section{Remarks:}

(i) The role of the local output is to compensate the discrete-type global output to produce a continuous final reasoning surface. The Dubois and Prade's parametric $t$ norm would yield various compensation behaviours by adjusting the $\alpha$ value.

(ii) The general equations listed above can be simplified by assigning special values to $\alpha$. If $\alpha=0$, the Dubois and Prade's parametric $t$-norm is then a standard fuzzy intersection (min), and the local nonlinear compensator

IEE Proc.-Control Theory Appl., Vol. 147, No. 2, March 2000 becomes

$\left(u_{D}^{*}\right)_{\text {mit }}=\left\{\begin{array}{ll}\frac{\mathcal{R}\left(U_{k+2}^{*}-U_{k+1}^{*}\right)-(1-\varepsilon)\left(U_{k+1}^{*}-U_{k}^{*}\right)}{1+2(1-\varepsilon)} & \text { region I } \\ \frac{\varepsilon\left(U_{k+2}^{*}-U_{k+1}^{*}\right)-(1-\mathcal{R})\left(U_{k+1}^{*}-U_{k}^{*}\right)}{1+2(1-\mathcal{R})} & \text { region II } \\ \frac{\varepsilon\left(U_{k+2}^{*}-U_{k+1}^{*}\right)-(1-\mathcal{R})\left(U_{k+1}^{*}-U_{k}^{*}\right)}{1+2 \varepsilon} & \text { region III } \\ \frac{\mathcal{R}\left(U_{k+2}^{*}-U_{k+1}^{*}\right)-(1-\varepsilon)\left(U_{k+1}^{*}-U_{k}^{*}\right)}{1+2 \mathcal{R}} & \text { region IV }\end{array}\right.$. 

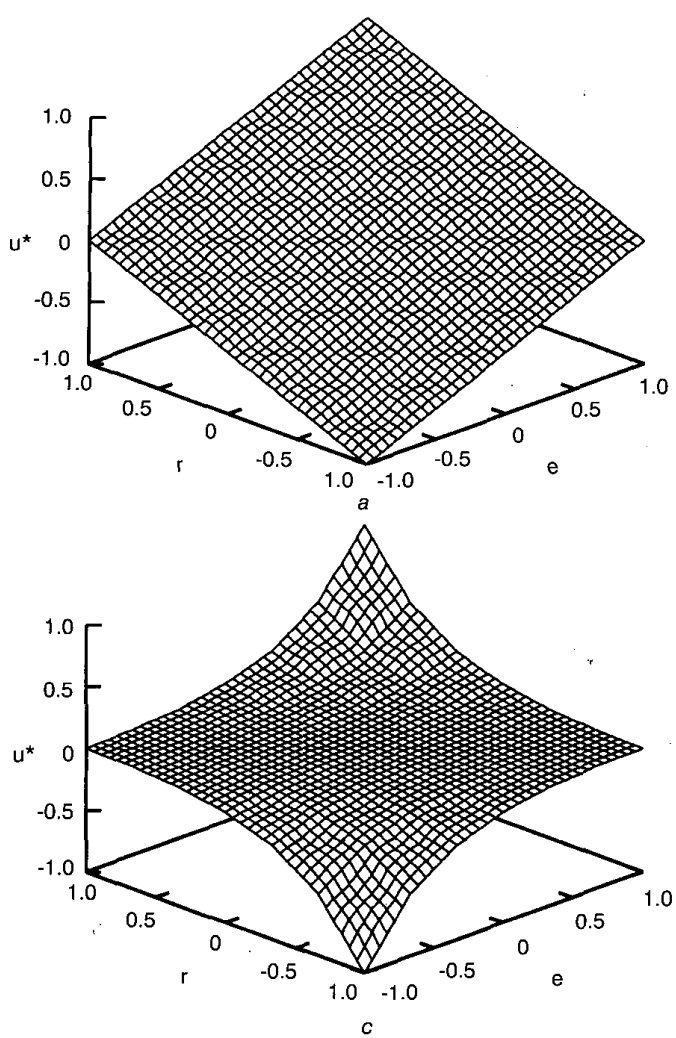
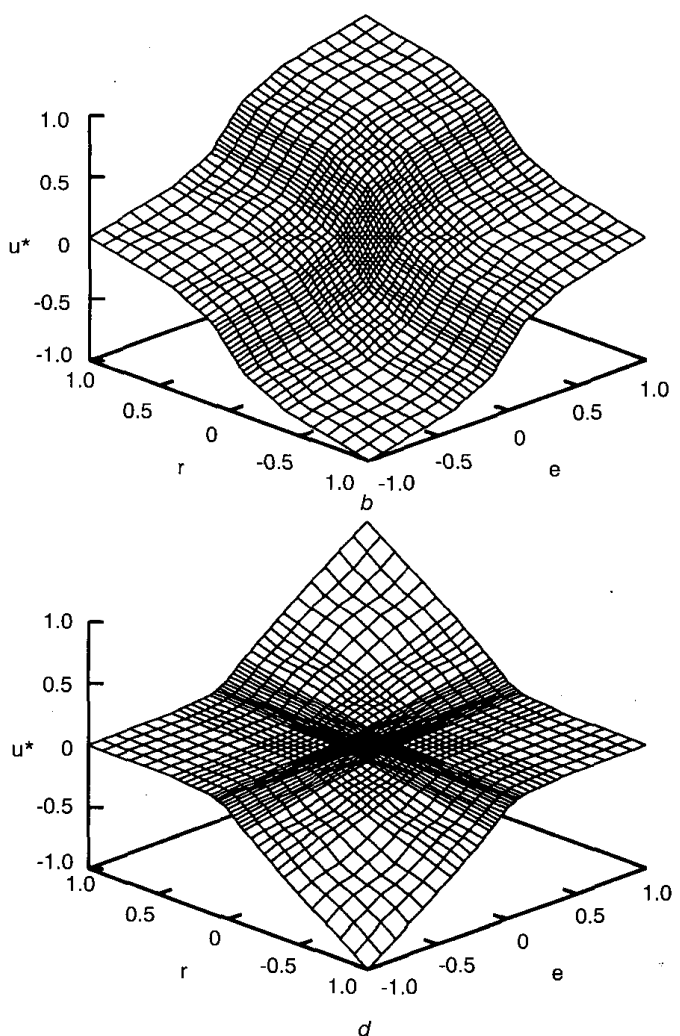

Fig. 7 Effects of shrinking factors on final reasoning surface of the crisp-type SSMFs-FLC $m_{e}=m_{r}=3, \alpha=0.5$ in Dubois and Prade's parametric t-norm $a s_{\mathrm{e}}=s_{r}=1.0, s_{u}=1.0$ $a s_{\mathrm{e}}=s_{r}=1.0, s_{u}=1.0$
$s_{\mathrm{e}}=s_{r}=0.6, s_{u}=1.0$ $c s_{\mathrm{e}}=s_{r}=1.0, s_{u}=0.6$ $d s_{\mathrm{e}}=s_{r}=0.6, s_{u}=0.6$

If $\alpha=1$, the Dubois and Prade's parametric $t$-norm is then an algebraic product, and the local nonlinear compensator becomes

$$
\begin{aligned}
\left(u_{D}^{*}\right)_{d p}= & (\varepsilon)(\mathcal{R})\left(U_{k+2}^{*}-U_{k+1}^{*}\right) \\
& -(1-\varepsilon)(1-\mathcal{R})\left(U_{k+1}^{*}-\dot{U}_{k}^{*}\right)
\end{aligned}
$$

(iii) The effects of distributions of principal values for $e, r$ and $u$ on final reasoning surface, for both global and local parts, can be further investigated. Without a loss of generality, only the SSMFs-FLC and ESMFs-FLC are illustrated in the following. For input variables of the SSMFs-FLC, the values of $s_{e}$ and $s_{r}$ influence the location and the shape of the $i j$ th block in the domain $U_{e} \times U_{r}$. In light of this, a specific set of $\left(e^{*}, r^{*}\right)$ may be located in a different $i j$ th block for different $s_{e}$ and $s_{r}$ values and, thus, may instigate distinct control rules in obtaining the final output. Regarding the output variable $u$, different effects are induced for $u_{G}^{*}$ and $u_{L}^{*}$ by changing the shrinking factor $s_{u}$. For $u_{G}^{*}$, modifying $s_{u}$ only changes its magnitude. However, for $u_{L}^{*}$, both magnitude and nonlinearity are altered by varying the value of $s_{u}$.

Fig. 5 shows the $t$-norm-independent global response for the crisp-type SSMFs-FLCs with various shrinking factors. Figs. 6 illustrates the influence of $t$-norms on local outputs. Fig. 7 illustrates the effects of the shrinking factors for $\alpha=0.5$ in the Dubois and Prade's parametric $t$-norm on the final reasoning surfaces.

\section{Conclusion}

This study has examined the input/output parametric relationship of a class of crisp-type fuzzy logic controllers using the Dubois and Prade's parametric $t$-norm-sum-gravity inference method. The topology of the FLCs being studied is defined as follows: (a) the input membership functions are composed of a series of unequally spaced triangles and the output membership functions consist of a set of singletons (the crisp type); $(b)$ the Dubois and Prade's parametric $t$-norm is used in calculating the firing level and implication of each single control rule; $(c)$ the inference results of all control rules are summed together to produce a final output fuzzy set; and $(d)$ the centre-ofgravity method is used for defuzzification. The explicit mathematical expressions of reasoning surfaces for the FLCs, using different $\alpha$ values of the Dubois and Prade's parametric $t$-norm as the firing operator, are addressed. The reasoning outputs of these FLCs are decomposed into two parts: a two-dimensional multilevel relay which is independent of the $t$-norm used and a local nonlinear compensator with different output patterns according to the $\alpha$ value of the Dubois and Prade's parametric $t$-norm. Such theoretical analysis is significant for illustrating the inner characteristics of fuzzy logic controllers.

\section{Acknowledgments}

The authors would like to thank the National Science Council of the Republic of China for financially supporting this research under Contract NSC87-2214-E-002-014. 


\section{References}

1 ZADEH, L.A.: 'Fuzzy sets', Inf. Control, 1965, 8, pp. 338-353

2 MAMDANI, E.H.: "Applications of fuzzy algorithms for simple dynamic plant', IEE, 1974, 121, pp. 1585-1588

3 LEE, C.C.: 'Fuzzy logic in control systems: fuzzy logic controller, Part I and II', IEEE Trans. Syst. Man Cybern., 1990, SMC-20, pp. 404-435

4 CHEN, C.-L. WANG, S.-N., HSIEH, C.-T., and CHANG, F.-Y. 'Theoretical analysis of the crisp-type fuzzy logic controllers using various-norm-sum-gravity inference methods', IEEE Trans. Fuzzy Syst, 1998, 6, pp. 122-136

5 NGUYEN, H.T., SUGENO, M., TONG, R., and YAGER, R.R.: 'Theoretical aspects of fuzzy control' (John Wiley \& Sons. Inc., New York, 1995)

6 SILER, W., and YING, H.: 'Fuzzy control theory: the linear case', Fuzzy Sets Syst., 1989, 33, pp. 275-290

7 MALKI, H., LI, H., and CHEN, G.: 'New design and stability analysis of fuzzy proportional-derivative control systems', IEEE Trans. Fuzzy Syst., 1994,2 , pp. $245-254$

8 TANAKA, K., and SUGENO, M.: 'Stability analysis and design of fuzzy control systems', Fuzzy Sets Syst., 1992, 4, pp. 135-156

9 WANG, H.O., TANAKA, K., and GRIFFIN, M.F.: 'An approach to fuzzy control of nonlinear systems: stability and design issues', IEEE Trans. Fuzzy Syst., 1996, 4, pp. 14-23
10 CHEN, C.-L., and KUO, F.-C.: 'Design and analysis of a fuzzy logic controller', Int. J. Syst. Sci., 1995, 26, pp. 1223-1248

11 LEWIS, F.L., and LIU, K.: 'Towards a paradigm for fuzzy logic control', Automatica, 1996, 32, pp. 167-181

12 QIAO, W., and MIZUMOTO, M.: 'PID type fuzzy controller and parameters adaptive method', Fuzzy Sets Syst., 1996, 78, pp. 23 35

13 YING, H.: 'A nonlinear fuzzy controller with linear control rules is the sum of a global two-dimensional multilevel relay and a local nonlinear proportional-integral controller', Automatica, 1993, 29, pp. 499-505

14 YING, H.: 'General analytical structure of typical fuzzy controller and their limiting structure theorems', Automatica., 1993, 29, pp. 1139-1143

15 YING, H.: 'The simplest fuzzy controllers using different inference YING, H.: 'The simplest fuzzy controllers using different inference
methods are different nonlinear proportional-integral controllers with methods are different nonlinear proportional-integral
variable gains', Automatica., 1993, 29, pp. 1579-1589

16 CHEN, C.-L., WANG, S.-N., HSIEH, C.-T., and CHANG, F.-Y. Theoretical analysis of the FLC with unequally-spaced triangular membership functions', Fuzzy Sets Syst., 1999, 101, pp. 87-108

17 CHEN, C.-L. and HSIEH, C.-T.: 'User-friendly design method for fuzzy logic controller', IEE Proc., Control Theory Appl., 1996, 143, pp. 358366

18 KLIR, G.J., and YUAN, B.: 'Fuzzy sets and fuzzy logic: theory and applications' (Prentice Hall International, 1995) 